\title{
The role of the oceans in shaping the tropospheric response to the 11 year solar cycle
}

\author{
Stergios Misios ${ }^{1,2}$ and Hauke Schmidt ${ }^{2}$ \\ Received 22 October 2013; revised 23 November 2013; accepted 25 November 2013; published 17 December 2013.
}

[1] Observational data indicate a weakening and poleward shift of the subtropical tropospheric jets in the maximum phase of the 11 year solar cycle, commonly explained in terms of a direct "top-down" propagation of solar signals from the stratosphere to the troposphere. We here demonstrate possible linkages to oceanic variability, instead. The observed response of the jets is qualitatively and quantitatively reproduced in an ensemble of simulations with a global model forced only at the lower boundary by the observed sea surface temperatures and sea ice concentrations, while keeping solar cycle forcing constant. The twentieth century reanalysis, in which only surface observations are assimilated, is characterized by a similar shift of the jets. These findings suggest that changes at the ocean surface could contribute considerably to the poleward shift of the subtropical tropospheric jets, although a topdown influence on the oceans and hence indirectly on the jets cannot be excluded. Citation: Misios, S., and H. Schmidt (2013), The role of the oceans in shaping the tropospheric response to the 11 year solar cycle Geophys. Res. Lett., 40, 6373-6377, doi:10.1002/2013GL058439.

\section{Introduction}

[2] Variations of the solar irradiance in the course of the 11 year solar cycle, both total and spectral, may alter tropospheric circulation. There have been observational indications for a weakening and poleward shift of the subtropical tropospheric jets, a poleward shift of the Hadley circulation, a stronger Walker circulation together with excess precipitation over the convergence zones, and a warmer troposphere in solar maximum [e.g., Haigh et al., 2005; Labitzke and van Loon, 1995; Gleisner and Thejll, 2003; van Loon et al., 2007]. If of genuine solar origin, the amplitude and spatial patterns of the observed responses cannot be explained easily by the miniscule increase in the direct radiative heating of the troposphere in solar maximum [Larkin et al., 2000]. Amplification mechanisms need to be involved, instead. Earlier studies proposed various such mechanisms which are, in general, falling into two separate broad categories [Gray et al., 2010]. In the first category of "top-down" mechanisms (TDMs), it is assumed that strong solar signals in the troposphere are brought about from the stratosphere via

\footnotetext{
Additional supporting information may be found in the online version of this article.

${ }^{1}$ Laboratory of Atmospheric Physics, Aristotle University of Thessaloniki, Thessaloniki, Greece.

${ }^{2}$ Max Planck Institute for Meteorology, Hamburg, Germany.

Corresponding author: S. Misios, Laboratory of Atmospheric Physics, Aristotle University of Thessaloniki, Thessaloniki, Greece. (misios@auth.gr)

(C)2013. American Geophysical Union. All Rights Reserved. 0094-8276/13/10.1002/2013GL058439
}

either a polar or an equatorial route. Enhanced ultraviolet radiation and the ozone feedback warm the upper stratosphere and lead to zonal wind changes, which can influence the propagation of planetary waves, particularly in boreal winter. This synergy between radiation, chemistry, and dynamics could propagate solar signals from the stratosphere downward to the troposphere [Kodera and Kuroda, 2002]. One further consequence of this mechanism is that a weaker Brewer-Dobson circulation, a reduced tropical upwelling, and hence an anomalous warming in the tropical lower stratosphere (TLS) should be expected in solar maximum. Haigh et al. [2005] proposed an equatorial TDM by which a warmer TLS and the associated changes in the eddy momentum deposition could explain the observational notion of a poleward shift of the subtropical tropospheric jets. In contrast, the second category of "bottom-up" mechanisms (BUMs) postulates that solar signals in the troposphere originate from the oceans. For example, Meehl et al. [2008] proposed that enhanced absorption of visible solar radiation by the oceans, air-sea coupling, and cloud feedbacks could intensify large-scale circulation systems of the troposphere such as the Walker and Hadley cells. The troposphere, therefore, could be sensitive to solar signals both from the stratosphere and oceans and synergistic effects of TDMs and BUMs could amplify or diminish the response.

[3] Most of the past studies modeled effects of solar spectral variability on the stratosphere and a top-down propagation into the troposphere, neglecting ocean changes [e.g., Matthes et al., 2006; Schmidt et al., 2010]. The relative role of the oceans in carrying solar signals into the troposphere has not been examined in great detail. We here examine whether the observed ocean surface variability, possibly due to TDMs and BUMs, can explain some of the tropospheric responses to solar cycle such as the poleward shift of the subtropical jets. To give a plausible answer, we compare solar signals in reanalysis data, in which the troposphere responds both to stratospheric and oceanic perturbations, and tailored model simulations, in which the direct forcing from the stratosphere is suppressed.

\section{Selection of Data Sets to Isolate Mechanisms}

[4] The first data set used in this study is the latest interim reanalysis (ERA-int) from the ECMWF (European Centre for Medium-Range Weather Forecasts), available in global latitude/longitude grids of $1.5 \times 1.5^{\circ}$ resolution, with 37 pressure levels up to $1 \mathrm{hPa}$ [Dee et al., 2011]. The chosen period is 1979 to 2009 , during which data quality is improved by assimilating satellite observations. ERA-int will only be used as a reference because pathways (i.e., TDM or BUM) leading to possible solar signals in the troposphere cannot be separated easily in this data set. 
[5] Ensemble simulations with chemistry-climate general circulation models forced with realistic spectral solar irradiance (SSI) variations and fixed (climatological) surface boundary conditions should isolate part of the signal coming directly from the stratosphere [Matthes et al., 2006; Schmidt et al., 2010]. On the other hand, to study how solar signals in the oceans influence the troposphere, one need to carry out simulations with a coupled atmosphere-ocean general circulation model (GCM) forced by total solar irradiance (TSI) changes alone [Meehl et al., 2008]; in this case, however, results would rely heavily upon the modeled response of the oceans, which at present remains uncertain [Gray et al., 2010]. Coordinated multimodel simulations could be a remedy but a much simpler option is to carry out ensemble simulations with an atmospheric GCM forced by time-varying observations of sea surface temperatures (SSTs) and sea ice concentrations (SICs) alone. At the same time, the solar forcing should be kept constant (i.e., constant TSI and SSI). Certainly, this methodology cannot replace coupled atmosphere-ocean GCM simulations but it allows testing the role of the observed ocean variability because in such a model setup, we know a priori that solar signals in the troposphere are brought about from the oceans. Hence, any direct influence of the stratosphere is suppressed.

[6] One option to test this alternative methodology is given by the twentieth century reanalysis [Compo et al., 2011]. The horizontal resolution of this data set (hereafter 20CR) is T62 with 24 pressure levels in the vertical up to $10 \mathrm{hPa}$. The $20 \mathrm{CR}$ has been produced from an ensemble of simulations with a global forecast model in which surface pressure observations are only assimilated. This differs from the ERA-int approach that additionally assimilates atmospheric observations (e.g., radiosondes, balloons, and satellites). Time-varying $\mathrm{CO}_{2}$ concentrations and volcanic aerosols are also considered by the 20CR forecast model, and observations of SST and SIC taken from the Met Office HadISST v.1.1 are prescribed at the lower boundary. The solar cycle variation is specified in terms of TSI, whereas SSI variability is neglected. The full data set spans from 1871 to 2010 but we here consider the subperiod 1979-2009.

[7] The assimilation of surface pressure observations in 20CR may introduce an unwanted top-down component, indirectly, contaminating results. This is because significant solar signals in surface pressure have been reported over the Northern Hemisphere (NH) [van Loon et al., 2007], the origin of which may be traced back to dynamical changes in the stratosphere [Ineson et al., 2011]. To alleviate this shortcoming, we additionally carried out an ensemble of 10 simulations (hereafter ENS) with the middle atmosphere version of European Centre/Hamburg GCM (MAECHAM5) [Manzini et al., 2006] forced only by the SST and SIC Met Office HadISST v.1.1 observations. All integrations have been carried out with constant (average solar activity) SSI and TSI and changes in greenhouse gases and volcanic aerosols are neglected. For computational efficiency and to allow for a sufficient ensemble size, the spectral model is used at a fairly coarse T31 triangular truncation (equivalent to $3.75^{\circ} \times 3.75^{\circ}$ grid) but with 90 pressure levels in the vertical up to $0.01 \mathrm{hPa}$. Simulations cover the period from 1952 to 2006, but only years after 1979 are analyzed for consistency with the other two data sets. ENS might serve as a better test bed for establishing a connection between solar signals in the troposphere and the oceans because in such a model configuration, solar signals in the troposphere can only be communicated from below. Even in ENS, however, an indirect forcing of the troposphere from stratospheric changes is still possible under the assumption that the observed SST variability contains a top-down forced component. This caveat will be further discussed in section 4 .

\section{Comparison of Solar Regression Coefficients}

[8] We calculate solar signals in deseasonalized monthly zonal-mean zonal winds $(\bar{u})$ and temperatures $(\bar{T})$ with an autoregressive (first-order) multiple linear regression model (MLR-AR1) that considers six standardized predictors: the atmospheric $\mathrm{CO}_{2}$ concentration, the optical depth of stratospheric aerosols primarily injected by major volcanic eruptions, the $10.7 \mathrm{~cm}$ solar flux $\left(F_{10.7}\right)$, the Niño-3.4 index (SST anomalies over $5^{\circ} \mathrm{S}-5^{\circ} \mathrm{N}, 120^{\circ} \mathrm{W}-170^{\circ} \mathrm{W}$ ) to capture the El Niño-Southern Oscillation (ENSO) signature, and two orthogonal indices to account for the quasi-biennial oscillation $(\mathrm{QBO}$ indices are the first and the second principal components of the zonal-mean zonal winds over $15^{\circ} \mathrm{S}$ $15^{\circ} \mathrm{N}$ and $70-5 \mathrm{hPa}$ ). QBO predictors are not considered in 20CR because QBO effects are not explicitly simulated (see supporting information Table S1). Statistical significance of the regression coefficients is examined with a standard two-tailed $t$ test with rejection level set to $95 \%$.

[9] Figure 1a displays the $\bar{u}$ regression coefficients onto $F_{10.7}$ for the ERA-int, scaled to 100 solar flux units (sfu) to be comparable with other studies. Similar to previous MLR analyses [e.g., Haigh et al., 2005; Crooks and Gray, 2005], the midlatitude response after 1979 is characterized by meridional dipoles of statistically significant negative/positive anomalies with amplitudes up to about $0.7 \mathrm{~m} / \mathrm{s} / 100 \mathrm{sfu}$. A comparison to the climatological zonal winds (contours) confirms the notion of weakened and poleward-shifted jets. In the equatorial troposphere, positive anomalies are detected but they do not exceed the $95 \%$ confidence level. According to the equatorial top-down mechanism of Haigh et al. [2005], the observed weakening and poleward displacement of the jets in solar maximum is associated to stability changes in TLS. ERA-int shows a significant warming in TLS with values up to $0.4 \mathrm{~K} / 100 \mathrm{sfu}$ (Figure 2, green line) consistent to other reanalysis products [e.g., Crooks and Gray, 2005]. A warmer TLS in solar maxima could result from a reduced upwelling in solar maximum as suggested by Kodera and Kuroda [2002]. Much stronger temperature anomalies on the order of $1 \mathrm{~K} / 100 \mathrm{sfu}$ are seen in the upper tropical stratosphere associated to increased ultraviolet radiation and ozone feedback. A weak and insignificant cooling is detected in the tropical middle stratosphere but its magnitude and the exact position varies among different reanalyses.

[10] Compared to ERA-int, the response of the tropical stratosphere to solar cycle should be weaker in 20CR because the stratospheric heating rates are calculated from a broadband parameterization [Compo et al., 2011] and hence reflect the weak variation of TSI $(\sim 0.1 \%)$, not SSI. The 20CR forecast model is also lacking a well-resolved stratosphere and hence the dynamical TDM of Kodera and Kuroda [2002], which involves wave-mean flow interactions, may not sufficiently be represented. For these reasons, solar-related temperature anomalies in the stratosphere in 

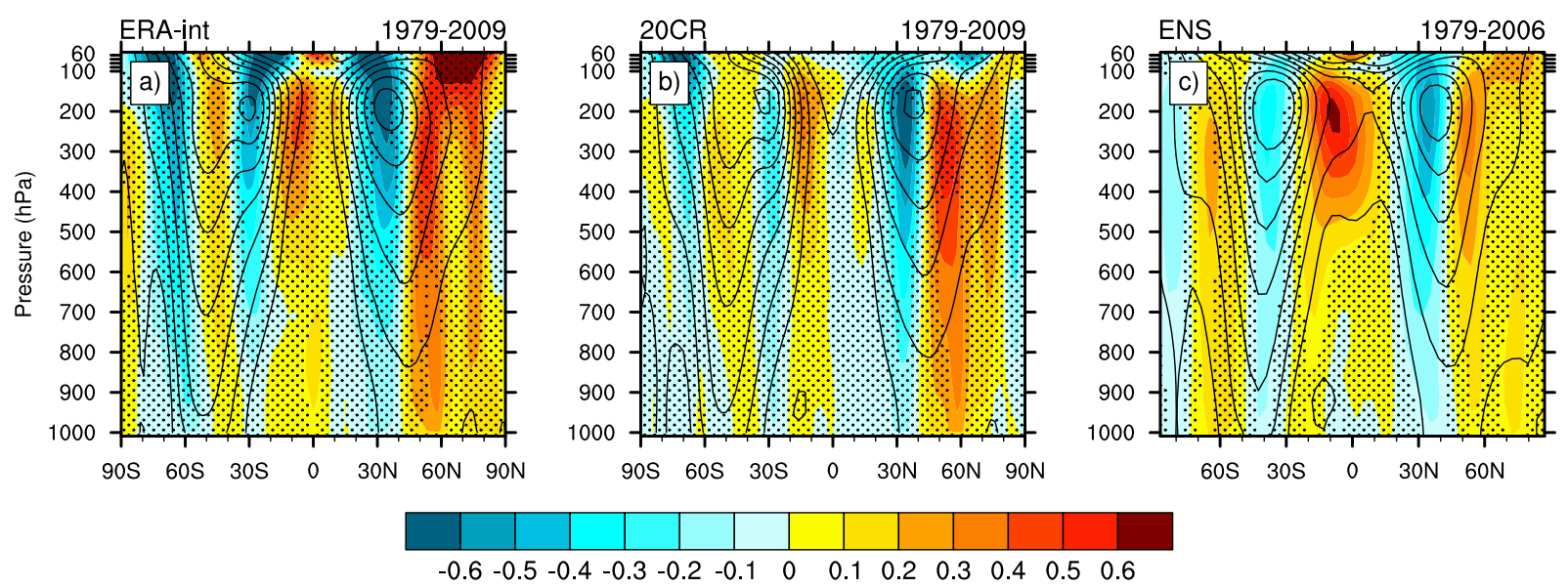

Figure 1. Regression coefficients of monthly $\bar{u}$ anomalies onto the solar $F_{10.7}$ flux (m/s/100 sfu) from (a) ERA-int, (b) 20CR, and (c) ENS. Shaded areas indicate statistical significance below $95 \%$ according to a Student's $t$ test.

general and the TLS in particular are expected to be weaker in $20 \mathrm{CR}$ compared to the reference data set. This is best demonstrated in Figure 2 (red line), which shows an almost 4 times weaker warming in TLS. If the observed shift of the tropospheric jets is primarily controlled by changes in TLS, as proposed by Haigh et al. [2005], much weaker responses should then be detected in 20CR, provided that the relationship is linear. Figure $1 \mathrm{~b}$, however, shows equally strong vertical bands of negative/positive $\bar{u}$ anomalies in both hemispheres. The position of the $\bar{u}$ dipoles indicates a weakening and a poleward shift of the subtropical jets in solar maximum, in a fashion quantitatively and qualitatively similar to ERA-int. This suggests that the observed circulation changes in the troposphere can be produced independently of a strong TLS warming and gives evidence for connections to the surface variability. In $20 \mathrm{CR}$, the surface variability is constrained by the specification of historical SSTs and SICs and the assimilation of pressure observations. The latter, as

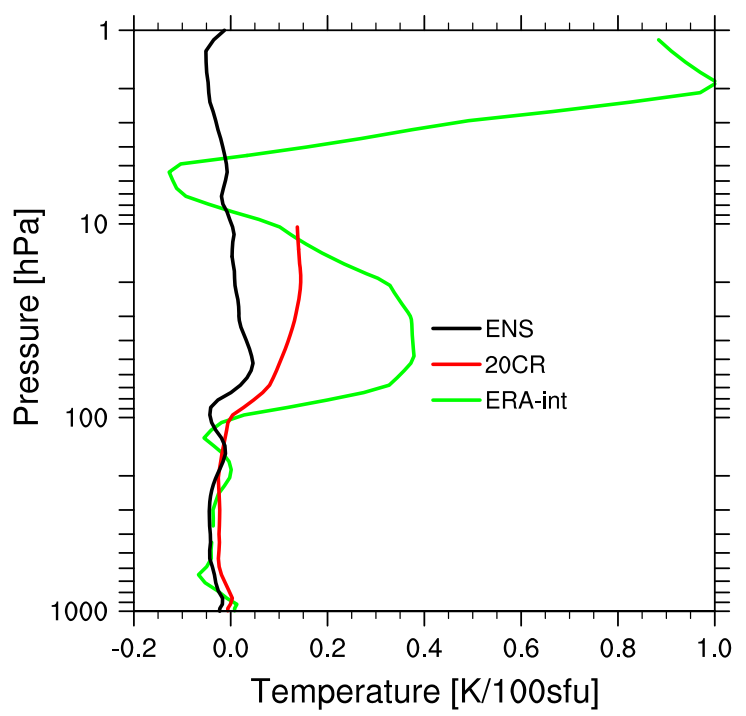

Figure 2. Regression coefficients of monthly $\bar{T}$ anomalies averaged over the Tropics $\left(25^{\circ} \mathrm{S}-25^{\circ} \mathrm{N}\right)$ onto the solar $F_{10.7}$ flux (K/100 sfu) from (a) ERA-int (green), (b) 20CR (red), and (c) ENS (black). mentioned earlier, could introduce a top-down component, indirectly. As such, a poleward shift of the jets in 20CR could actually reflect changes in the stratosphere, introduced by the assimilation of pressure observations. The following analysis of ENS reduces this possibility as it provides a much clearer case to demonstrate linkages to ocean surface temperature variability.

[11] As expected from a constant TSI and SSI forcing in ENS, the simulated temperature anomalies in the tropical stratosphere do not differ, statistically, from zero (Figure 2, black line). Yet dipoles of negative/positive $\bar{u}$ anomalies on the order of $0.3 \mathrm{~m} / \mathrm{s} / 100 \mathrm{sfu}$ are simulated in the troposphere (Figure 1c). Statistical significance is obtained in large parts of the troposphere, implying a robust response among individual ensemble members (see below). As in the other two data sets, increased solar activity is associated with a poleward shift of the NH subtropical jet. Poleward of $45^{\circ} \mathrm{N}$, positive anomalies throughout the depth of the troposphere are seen in all data sets. In the equatorial upper troposphere, positive zonal wind anomalies of about $0.5 \mathrm{~m} / \mathrm{s} / 100 \mathrm{sfu}$ are simulated while further southward, a streak of negative anomalies is positioned at the core of the SH jet. This is in contrast to the $\bar{u}$ anomalies seen in the other two data sets. Associated to wind changes, vertical columns of significant positive temperature anomalies are identified in the $\mathrm{NH}$ midlatitude troposphere in all data sets (supporting information Figure S1). Haigh [2003] detected similar vertical columns of warming and the analysis of Zhou and Tung [2013] over the extended period 1955-2011 showed that they can be seen in all seasons, independently of thermal changes in TLS. This further supports our argument that the observed circulation changes in the troposphere, and particularly, a poleward shift of the jets may be related to oceanic variability.

[12] To study the intraensemble variability in ENS of the jet response to the solar cycle, Figure 3 shows all $\bar{u}$ regression coefficients averaged over 100 to $200 \mathrm{hPa}$ in every individual ensemble member (thin black lines). This band has been chosen to characterize responses in the upper troposphere, where robust signals appear. Regression coefficients from ERA-int and 20CR paired with their uncertainties (one standard deviation) are also shown. Note that both the amplitude and the observed latitudinal variation between about $10^{\circ} \mathrm{S}$ and $60^{\circ} \mathrm{N}$ seen in ERA-int is captured in 7 out of the 


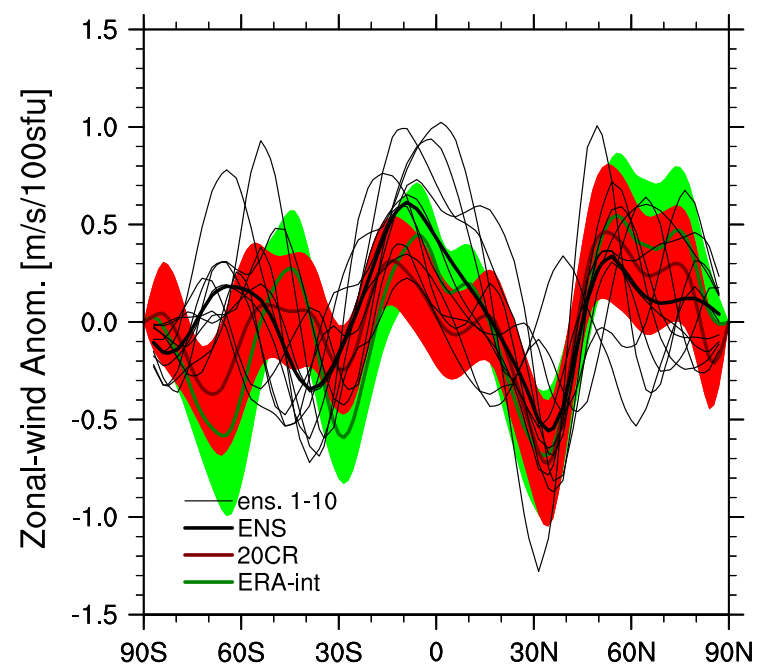

Figure 3. Latitudinal profiles of the $\bar{u}$ regression coefficients onto the solar $F_{10.7}$ flux (m/s/100 sfu) averaged from 100 to $200 \mathrm{hPa}$ from ERA-int (green line), 20CR (red line), and ENS (thin black lines). The green and red shading indicate 1 standard deviation confidence levels. The thick black line is the ensemble mean average of ENS.

10 ensemble members. Farther north, the signal is more variable, but the ensemble mean response tracks reasonably well the meridional profiles of ERA-int and 20CR. Likewise, signals in 20CR follow closely those in the reference data set. The resemblance between the ENS and ERA-int weakens in the Southern Hemisphere, where the simulated subtropical minimum of $\bar{u}$ anomalies is somewhat shifted to higher latitudes. This inconsistency, however, is likely related to the unrealistic position and shape of the $\mathrm{SH}$ jet in MAECHAM5 (contours in Figure 1c). Our model in the chosen horizontal resolution (T31) produces a too narrow SH jet with maximum winds located poleward to the jet core in ERA-int $\left(40^{\circ} \mathrm{S}\right.$ in MAECHAM5 versus $30^{\circ} \mathrm{S}$ in ERA-int), whereas the position of the $\mathrm{NH}$ jet is realistic. Same reasoning could explain why 20CR captures very well the poleward shift of the jets in both hemispheres.

\section{Discussion and Conclusions}

[13] Solar signals in the troposphere have often been explained in terms of a direct forcing from the stratosphere. Earlier model simulations, without ocean coupling, provided physical linkages between a UV heating of the stratosphere, a weakening of the Brewer-Dobson circulation, an adiabatic warming of the TLS, changes in the eddy momentum deposition, and a weakening and poleward shift of the subtropical tropospheric jets in solar maxima, as shown in Figure 1a [Kodera and Kuroda, 2002; Haigh et al., 2005; Matthes et al., 2006; Schmidt et al., 2010]. The fact that coupled atmosphere-ocean GCMs forced only with TSI variability (i.e., TDMs switched off) failed to reproduce solar signals of realistic amplitudes [Meehl et al., 2008] provides further evidence on the importance of the stratosphere. In some atmosphere-ocean simulations, however, the strength, the effectiveness, or the very nature of a bottomup forcing itself may have been misrepresented [Stott et al., 2003]. For instance, the aqua-planet simulations of Cai and Tung [2012] indicated that the extra water vapor in solar maximum may be transported to the upper tropical troposphere, not to the convective zones as in the BUM of Meehl et al. [2008], where the anomalous heating due to latent heat release induces large-scale circulation changes, characterized by a poleward shift of the subtropical tropospheric jets. This mechanism, however, has not been tested within an atmosphere-ocean GCM framework.

[14] Our analysis shows that a strong warming in the TLS may not be a prerequisite for simulating a poleward shift of the tropospheric subtropical jets. Despite the fact that the TLS warms too little in 20CR or not at all in ENS, the $\mathrm{NH}$ jet weakens and shifts poleward in a fashion qualitatively and quantitatively similar to ERA-int (Figures 1 and 2). A displacement of the $\mathrm{SH}$ jet is realistically captured in 20CR only. The equatorial top-down mechanism proposed by Haigh et al. [2005], therefore, may not be the only possibility to explain the observed circulation changes in the troposphere. The deliberately simplified framework of ENS points to the ocean surface variability as an alternative explanation. In fact, a poleward shift of the jets appears to be a characteristic response to different types of forcings such as polar ozone depletion that cools the lower stratosphere or greenhouse gas increase that warms the upper troposphere or the negative phase of ENSO [e.g., Son et al., 2008; Seager et al., 2003]. A possible aliasing in observations between signals of the above mentioned forcings and the solar cycle cannot be excluded in ERA-int and 20CR but the analysis of ENS runs weakens such a possibility. An ENSOsolar cycle contamination is still possible in ENS (as well as in ERA-int and 20CR) given the shortness of the period under investigation. A separate analysis over an extended period (after 1955) identifies a similar poleward shift of the subtropical jets, albeit of somewhat weaker amplitude (supporting information Figure S2). Moreover, solar cycle signals remain essentially unchanged when the Niño-3.4 index is taken out of the MLR-AR1 model, further reducing the possibility of an ENSO-solar cycle contamination.

[15] Although ENS underlines the role of oceans in controlling tropospheric responses to the solar cycle, the specification of surface observations does not automatically allow us to exclude an indirect influence from TDMs, as they could produce changes first at the ocean surface, perhaps together with BUMs, and then in the troposphere. A possible sequence could involve a perturbation of the surface pressure at the $\mathrm{NH}$ [van Loon et al., 2007; Ineson et al., 2011] from the polar route TDM with ensuing circulation changes, which could be amplified and extended to lower latitudes via air-sea coupling. In such a case, solar cycle signals seen in Figure 1c could actually be caused by stratospheric changes first affecting the surface. A similar mechanism could also explain signals in 20CR. Even if one assumes that the oceanic response to the solar cycle is controlled primarily by BUMs, their strength could depend or be modified by TDMs. This would mean that solar signals at the ocean surface could be brought about from a synergy of TDMs and BUMs and then affecting tropospheric circulation. These theoretical considerations have not been studied extensively, yet, and our study is not suited for this. Results from existing model simulations cannot be considered conclusive, either. Petrick et al. [2012] simulated oceanic responses to TDMs by using an ocean GCM forced by input taken from an atmospheric GCM integration simulating TSI and SSI effects. Although the demonstrated SST responses to the 11 year 
solar cycle forcing were weak, results should be interpreted with caution because the atmospheric GCM was not interactively coupled to an ocean model. As such, any putative top-down forcing of the oceans may be artificially dampened if atmosphere-ocean coupling amplifies the influence of TDMs.

[16] The previous discussion assumes that the specified SST observations contain a genuine 11 year solar cycle component, which in turn alters tropospheric circulation and moves subtropical jets toward the poles. However, the response of the oceans to the solar cycle is under debate. Modeling provided some evidence for solar cycle effects on the tropical oceans but failed to simulate consistent responses as cold or warm anomalies with increased solar activity were reported [Meehl et al., 2009; Misios and Schmidt, 2012]. Analyses of observations do not give consistent responses, either. van Loon et al. [2007] isolated a strong La Niña-like cooling in the tropical Pacific in peak years of solar activity, whereas the analysis of White et al. [1997] identified positive SST anomalies with El Niño-like spatial characteristics. Multiple regression analysis over the second part of the twentieth century showed an anomalous warming in the tropical Pacific in solar maximum [Roy and Haigh, 2010], a condition that should have favored the contracted subtropical jets [Seager et al., 2003]. Yet Figure 1 shows an extension in the opposite direction. An MLR-AR1 analysis of the SSTs over the period 1979-2009 does not reproduce the warming found by Roy and Haigh [2010] (supporting information Figure S3). Instead, the tropical Pacific is characterized by weak negative anomalies, which could cause a poleward shift of the jets as seen in Figure 1. Further work is necessary to resolve the apparent inconsistency between the observed signals on the surface and tropospheric circulation over the period after 1955. Furthermore, coordinated multimodel studies forced by either UV or visible variability will be needed to constrain TDM and BUM effects on oceanic variability and their possible extension into the troposphere.

[17] To summarize, we demonstrated a weakening and poleward shift of the NH tropospheric jet, as seen in observations after 1979, with a global model forced only at the lower boundary by SST and SIC observations. The twentieth century reanalysis, in which only surface observations are assimilated, shows a poleward shift of the jets in solar maximum in both hemispheres, consistent to solar signals in ERA-int. These findings suggest that oceans could act as a mediator of the solar cycle forcing into the troposphere but definite conclusions cannot be reached, before the role of TDMs and BUMs in producing oceanic variability is fully understood.

[18] Acknowledgments. This study was funded by the Deutsche Forschungsgemeinschaft (DFG) with the ARTOS project. We thank the Deutsches Klimarechenzentrum (DKRZ) for providing computational support. The authors thank two anonymous reviewers for constructive comments, which contributed significantly to improve the paper.

[19] The Editor thanks two anonymous reviewers for their assistance in evaluating this paper.

\section{References}

Cai, M., and K. K. Tung (2012), Robustness of dynamical feedbacks from radiative forcing: $2 \%$ solar versus $2 \times \mathrm{CO}_{2}$ experiments in an idealized GCM, J. Atmos. Sci., 69(7), 2256-2271, doi:10.1175/JAS-D-11-0117.1.

Compo, G. P., et al. (2011), The twentieth century reanalysis project, $Q . J$. R. Meteorolog. Soc., 137(654), 1-28, doi:10.1002/Qj.776.
Crooks, S. A., and L. J. Gray (2005), Characterization of the 11-year solar signal using a multiple regression analysis of the ERA-40 dataset, $J$ Clim., 18(7), 996-1015, doi:10.1175/JCLI-3308.1.

Dee, D. P., et al. (2011), The ERA-Interim reanalysis: Configuration and performance of the data assimilation system, Q. J. R. Meteorolog. Soc., 137(656), 553-597, doi:10.1002/Qj.828.

Gleisner, H., and P. Thejll (2003), Patterns of tropospheric response to solar variability, Geophys. Res. Lett., 30 (13), 44-1, doi:10.1029/ 2003 GL017129.

Gray, L. J., et al. (2010), Solar influences on climate, Rev. Geophys., 48, RG4001, doi:10.1029/2009RG000282.

Haigh, J. D. (2003), The effects of solar variability on the Earth's climate, Philos. Trans. R. Soc. London, Ser. A-Math. Phys. Eng. Sci., 361(1802), 95-111.

Haigh, J. D., M. Blackburn, and R. Day (2005), The response of tropospheric circulation to perturbations in lower-stratospheric temperature, $J$ Clim., 18(17), 3672-3685, doi:10.1175/JCLI3472.1.

Ineson, S., A. A. Scaife, J. R. Knight, J. C. Manners, N. J. Dunstone, L. J. Gray, and J. D. Haigh (2011), Solar forcing of winter climate variability in the Northern Hemisphere, Nat. Geosci., 4(11), 753-757, doi:10.1038/NGEO1282.

Kodera, K., and Y. Kuroda (2002), Dynamical response to the solar cycle, J. Geophys. Res., 107(D24), 4749, doi:10.1029/2002JD002224.

Labitzke, K., and H. van Loon (1995), Connection between the troposphere and stratosphere on a decadal scale, Tellus Ser. A-Dyn. Meteorol. Oceanogr., 47(2), 275-286, doi:10.1034/j.1600-0870.1995.t01-100008.x.

Larkin, A., J. D. Haigh, and S. Djavidnia (2000), The effect of solar UV irradiance variations on the Earth's atmosphere, Space Sci. Rev., 94(1-2), 199-214.

Manzini, E., M. A. Giorgetta, M. Esch, L. Kornblueh, and E. Roeckner (2006), The influence of sea surface temperatures on the northern winter stratosphere: Ensemble simulations with the MAECHAM5 model, $J$. Clim., 19(16), 3863-3881, doi:10.1175/JCLI3826.1.

Matthes, K., Y. Kuroda, K. Kodera, and U. Langematz (2006), Transfer of the solar signal from the stratosphere to the troposphere: Northern winter, J. Geophys. Res., 111, D06108, doi:10.1029/2005JD006283.

Meehl, G. A., J. M. Arblaster, G. Branstator, and H. van Loon (2008), A coupled air-sea response mechanism to solar forcing in the Pacific region, J. Clim., 21 (12), 2883-2897, doi:10.1175/ 2007JCLI1776.1.

Meehl, G. A., J. M. Arblaster, K. Matthes, F. Sassi, and H. van Loon (2009), Amplifying the Pacific climate system response to a small 11year solar cycle forcing, Science, 325(5944), 1114-1118, doi:10.1126/ science. 1172872 .

Misios, S., and H. Schmidt (2012), Mechanisms involved in the amplification of the 11-yr solar cycle signal in the tropical Pacific Ocean, J. Clim., 25(14), 5102-5118, doi:10.1175/Jcli-D-11-00261.1.

Petrick, C., K. Matthes, H. Dobslaw, and M. Thomas (2012), Impact of the solar cycle and the QBO on the atmosphere and the ocean, J. Geophys. Res., 117, D17111, doi:10.1029/2011JD017390.

Roy, I., and J. D. Haigh (2010), Solar cycle signals in sea level pressure and sea surface temperature, Atmos. Chem. Phys., 10(6), 3147-3153, doi:10.5194/acpd-9-25839-2009.

Schmidt, H., G. P. Brasseur, and M. A. Giorgetta (2010), Solar cycle signal in a general circulation and chemistry model with internally generated quasi-biennial oscillation, J. Geophys. Res., 115, D00I14, doi:10.1029/2009JD012542.

Seager, R., N. Harnik, Y. Kushnir, W. Robinson, and J. Miller (2003), Mechanisms of hemispherically symmetric climate variability, J. Clim., 16(18), 2960-2978, doi:10.1175/1520-0442(2003)016<2960:Mohscv >2.0.Co;2.

Son, S. W., et al. (2008), The impact of stratospheric ozone recovery on the Southern Hemisphere westerly jet, Science, 320(5882), 1486-1489, doi:10.1126/science.1155939.

Stott, P. A., G. S. Jones, and J. F. B. Mitchell (2003), Do models underestimate the solar contribution to recent climate change? J. Clim., 16(24), 4079-4093, doi:10.1175/1520-0442(2003)016<4079: DMUTSC $>2.0 . \mathrm{CO} ; 2$

van Loon, H., G. A. Meehl, and D. J. Shea (2007), Coupled air-sea response to solar forcing in the Pacific region during northern winter, J. Geophys. Res., 112, D02108, doi:10.1029/2006JD007378.

White, W., J. Lean, D. R. Cayan, and M. D. Dettinger (1997), Response of global upper ocean temperature to changing solar irradiance, J. Geophys. Res. C: Oceans, 102(C2), 3255-3266, doi:10.1029/96JC03549.

Zhou, J. S., and K. K. Tung (2013), Observed tropospheric temperature response to $11-y r$ solar cycle and what it reveals about mechanisms, $J$. Atmos. Sci., 70(1), 9-14, doi:10.1175/Jas-D-12-0214.1. 